\title{
On conformable delta fractional calculus on time scales
}

\author{
Dafang Zhao ${ }^{a}$, Tongxing $\mathrm{Li}^{\mathrm{b}, \mathrm{c}, *}$ \\ ${ }^{a}$ School of Mathematics and Statistics, Hubei Normal University, Huangshi, Hubei 435002, P. R. China. \\ ${ }^{b}$ LinDa Institute of Shandong Provincial Key Laboratory of Network Based Intelligent Computing, Linyi University, Linyi, \\ Shandong 276005, P. R. China. \\ 'School of Informatics, Linyi University, Linyi, Shandong 276005, P. R. China.
}

\begin{abstract}
In this paper, we introduce and investigate the concepts of conformable delta fractional derivative and conformable delta fractional integral on time scales. Basic properties of the theory are proved. (C)2016 All rights reserved.
\end{abstract}

Keywords: Conformable delta fractional derivative, conformable delta fractional integral, time scale.

2010 MSC: 26A33, 26E70.

\section{Introduction}

Fractional calculus is a generalization of ordinary differentiation and integration to arbitrary (non-integer) order. This subject is as old as the calculus of differentiation and goes back to times when Leibniz, Gauss, and Newton invented this kind of calculation. The fractional calculus always attracted interest of researchers due to its numerous applications in engineering, economics and finance, signal processing, dynamics of earthquakes, geology, probability and statistics, chemical engineering, physics, splines, thermodynamics, neural networks, and so on; see, for instance, the monographs by Carpinteri and Mainardi [19], Herrmann [20], Miller and Ross [26], Oldham and Spanier [27], Ortigueira [28], Podlubny [30], Sabatier et al. [31], Samko et al. [32], and the references cited therein.

Several definitions of a fractional derivative have been proposed. These definitions include Riemann-Liouville, Grunwald-Letnikov, Weyl, Caputo, Marchaud, and Riesz fractional derivatives.

${ }^{*}$ Corresponding author

Email addresses: dafangzhao@163.com (Dafang Zhao), litongx2007@163.com (Tongxing Li) 
However, nearly all fractional derivatives do not satisfy the well-known formula of the derivative of the product (the quotient) of two functions and the chain rule, etc. Recently, Khalil et al. [23] defined a new well-behaved simple fractional derivative which is called the "conformable fractional derivative" depending just on the basic limit definition of the derivative as follows: Let $f:[0,1] \rightarrow \mathbb{R}$ be a given function. Then for all $t>0$ and $\alpha \in(0,1)$, define $T_{\alpha}(f)$ by

$$
T_{\alpha}(f)(t):=\lim _{\varepsilon \rightarrow 0} \frac{f\left(t+\varepsilon t^{1-\alpha}\right)-f(t)}{\varepsilon},
$$

$T_{\alpha}(f)$ is termed the conformable fractional derivative of $f$ of order $\alpha$. By virtue of this definition, all the classical properties of the derivative hold; see [8, 22]. The notion of conformable fractional derivative was developed; we refer the reader to the papers [1, 6, 9, 10, 13] and the references cited there. In particular, Benkhettou et al. [13] introduced a conformable fractional calculus on an arbitrary time scale, which is a natural extension of the conformable fractional calculus.

A time scale $\mathbb{T}$ is an arbitrary nonempty closed subset of real numbers $\mathbb{R}$ with the subspace topology inherited from the standard topology of $\mathbb{R}$. The theory of time scales was born in 1988 with the Ph.D. thesis of Hilger [21]. The aim of this theory is to unify various definitions and results from the theories of discrete and continuous dynamical systems, and to extend such theories to more general classes of dynamical systems. It has been extensively studied on various aspects by several authors; see, for instance, the papers by [7, 11, 12, 14 18, 24, 25, 29].

In this paper, we introduce and investigate the concepts of conformable delta fractional derivative and conformable delta fractional integral on time scales which are different from those of [13]. This paper is organized as follows: Section 2 contains basic concepts of time scales. In Section 3 , the definition of conformable delta fractional derivative is introduced, and the basic properties of fractional derivative are investigated. In Section 4, we introduce and develop the notion of conformable delta fractional integral on time scales. We end Section 5 with conclusions and future research.

\section{Preliminaries}

Let $\mathbb{T}$ be a time scale. For $a, b \in \mathbb{T}$ we define the closed interval $[a, b]_{\mathbb{T}}$ by $[a, b]_{\mathbb{T}}:=\{t \in \mathbb{T}: a \leq$ $t \leq b\}$. The open and half-open intervals are defined in a similar way. For $t \in \mathbb{T}$ we define the forward jump operator $\sigma: \mathbb{T} \rightarrow \mathbb{T}$ and backward jump operator $\rho: \mathbb{T} \rightarrow \mathbb{T}$ by $\sigma(t):=\inf \{s \in \mathbb{T}: s>t\}$ and $\rho(t):=\sup \{s \in \mathbb{T}: s<t\}$, respectively, where $\inf \emptyset:=\sup \mathbb{T}$ and $\sup \emptyset:=\inf \mathbb{T}, \emptyset$ denotes the empty set.

Assume $t \in \mathbb{T}$. If $\sigma(t)>t$, then $t$ is right-scattered, and if $\rho(t)<t$, then $t$ is left-scattered. If $\sigma(t)=t$ and $t<\sup \mathbb{T}$, then $t$ is right-dense, while if $\rho(t)=t$ and $t>\inf \mathbb{T}$, then $t$ is left-dense. A point $t \in \mathbb{T}$ is dense if it is right-dense and left-dense at the same time; isolated if it is rightscattered and left-scattered at the same time. The forward graininess function $\mu: \mathbb{T} \rightarrow[0, \infty)$ and the backward graininess function $\eta: \mathbb{T} \rightarrow[0, \infty)$ are defined by $\mu(t):=\sigma(t)-t$ and $\eta(t):=t-\rho(t)$ for all $t \in \mathbb{T}$, respectively. If $\sup \mathbb{T}$ is finite and left-scattered, then $\mathbb{T}^{k}:=\mathbb{T} \backslash\{\sup \mathbb{T}\}$; otherwise, $\mathbb{T}^{k}:=\mathbb{T}$. If inf $\mathbb{T}$ is finite and right-scattered, then $\mathbb{T}_{k}:=\mathbb{T} \backslash\{\inf \mathbb{T}\}$; otherwise, $\mathbb{T}_{k}:=\mathbb{T}$. We set $\mathbb{T}_{k}^{k}:=\mathbb{T}^{k} \cap \mathbb{T}_{k}$.

A function $f: \mathbb{T} \rightarrow \mathbb{R}$ is called $r d$-continuous provided it is continuous at all right-dense points in $\mathbb{T}$ and its left-sided limits exist (finite) at all left-dense points in $\mathbb{T}$.

A function $f: \mathbb{T} \rightarrow \mathbb{R}$ is called regulated provided its right-sided limits exist (finite) at all right-dense points in $\mathbb{T}$ and its left-sided limits exist (finite) at all left-dense points in $\mathbb{T}$.

Assume $f: \mathbb{T} \rightarrow \mathbb{R}$ is a function and let $t \in \mathbb{T}^{k}$. Then we define $f^{\Delta}(t)$ to be the number (provided it exists) with the property that given any $\varepsilon>0$, there exists a neighborhood $U$ of $t$ such that

$$
\left|f(\sigma(t))-f(s)-f^{\Delta}(t)(\sigma(t)-s)\right| \leq \varepsilon|\sigma(t)-s|,
$$


for all $s \in U$. We call $f^{\Delta}(t)$ the delta derivative of $f$ at $t$ and we say that $f$ is delta differentiable on $\mathbb{T}^{k}$ provided $f^{\Delta}(t)$ exists for all $t \in \mathbb{T}^{k}$.

In what follows, let $\alpha \in(0,1]$.

\section{Conformable delta fractional derivative}

Definition 3.1. Assume $f: \mathbb{T} \rightarrow \mathbb{R}$ is a function and let $t \in \mathbb{T}^{k}$. Then we define $\mathbf{T}_{\alpha}\left(f^{\Delta}\right)(t)$ to be the number (provided it exists) with the property that given any $\varepsilon>0$, there exists a neighborhood $U$ of $t$ such that

$$
\left|(f(\sigma(t))-f(s)) \sigma^{1-\alpha}(t)-\mathbf{T}_{\alpha}\left(f^{\Delta}\right)(t)(\sigma(t)-s)\right| \leq \varepsilon|\sigma(t)-s|
$$

for all $s \in U$. We call $\mathbf{T}_{\alpha}\left(f^{\Delta}\right)(t)$ the conformable delta $(\Delta)$ fractional derivative of $f$ of order $\alpha$ at $t$. Moreover, we say that $f$ is conformable $\Delta$ fractional differentiable of order $\alpha$ on $t \in \mathbb{T}^{k}$ provided $\mathbf{T}_{\alpha}\left(f^{\Delta}\right)(t)$ exists for all $t \in \mathbb{T}^{k}$. The function $\mathbf{T}_{\alpha}\left(f^{\Delta}\right): \mathbb{T}^{k} \rightarrow \mathbb{R}$ is then called the conformable $\Delta$ fractional derivative of $f$ of order $\alpha$ on $\mathbb{T}^{k}$. We define the conformable $\Delta$ fractional derivative at 0 as

$$
\mathbf{T}_{\alpha}\left(f^{\Delta}\right)(0)=\lim _{t \rightarrow 0} \mathbf{T}_{\alpha}\left(f^{\Delta}\right)(t)
$$

If $t$ is right-dense, then we obtain the conformable fractional derivative reported in [13]. Some useful properties of conformable $\Delta$ fractional derivative of $f$ of order $\alpha$ are given in the following theorem.

Theorem 3.2. Let $\mathbb{T}$ be a time scale, $t \in \mathbb{T}^{k}$, and $\alpha \in(0,1]$. Then we have the following.

(i) If $f$ is conformable $\Delta$ fractional differentiable of order $\alpha$ at $t$, then $f$ is continuous at $t$.

(ii) If $f$ is continuous at $t$ and $t$ is right-scattered, then $f$ is conformable $\Delta$ fractional differentiable of order $\alpha$ at $t$ with

$$
\mathbf{T}_{\alpha}\left(f^{\Delta}\right)(t)=\frac{f(\sigma(t))-f(t)}{\mu(t)} \sigma^{1-\alpha}(t) .
$$

(iii) If $t$ is right-dense, then $f$ is conformable $\Delta$ fractional differentiable of order $\alpha$ at $t$ if and only if the limit

$$
\lim _{s \rightarrow t} \frac{f(t)-f(s)}{t-s} t^{1-\alpha}
$$

exists as a finite number. In this case,

$$
\mathbf{T}_{\alpha}\left(f^{\Delta}\right)(t)=\lim _{s \rightarrow t} \frac{f(t)-f(s)}{t-s} t^{1-\alpha} .
$$

(iv) If $f$ is conformable $\Delta$ fractional differentiable of order $\alpha$ at $t$, then

$$
f(\sigma(t))=f(t)+\mu(t) \mathbf{T}_{\alpha}\left(f^{\Delta}\right)(t) \sigma^{\alpha-1}(t) .
$$

Proof. Part (i). Assume that $f$ is conformable $\Delta$ fractional differentiable of order $\alpha$ at $t$. Then for each $\varepsilon>0$, there exists a neighborhood $U$ of $t$ such that

$$
\left|(f(\sigma(t))-f(s)) \sigma^{1-\alpha}(t)-\mathbf{T}_{\alpha}\left(f^{\Delta}\right)(t)(\sigma(t)-s)\right| \leq \varepsilon^{*}|\sigma(t)-s|
$$


for all $s \in U$, where

$$
\varepsilon^{*}=\frac{\varepsilon}{1+\left|\mathbf{T}_{\alpha}\left(f^{\Delta}\right)(t)\right|+2 \mu(t)}\left|\sigma^{1-\alpha}(t)\right| .
$$

Therefore, we have, for all $s \in U \cap\left(t-\varepsilon^{*}, t+\varepsilon^{*}\right)$,

$$
\begin{aligned}
|f(t)-f(s)|= & \mid f(\sigma(t))-f(s)-\mathbf{T}_{\alpha}\left(f^{\Delta}\right)(t)(\sigma(t)-s) \sigma^{\alpha-1}(t) \\
& -\left[f(\sigma(t))-f(t)-\mathbf{T}_{\alpha}\left(f^{\Delta}\right)(t)(\sigma(t)-t) \sigma^{\alpha-1}(t)\right]+\mathbf{T}_{\alpha}\left(f^{\Delta}\right)(t)(t-s) \sigma^{\alpha-1}(t) \mid \\
\leq & \varepsilon^{*}\left|(\sigma(t)-s) \sigma^{\alpha-1}(t)\right|+\varepsilon^{*}\left|(\sigma(t)-t) \sigma^{\alpha-1}(t)\right|+\left|\mathbf{T}_{\alpha}\left(f^{\Delta}\right)(t)(t-s) \sigma^{\alpha-1}(t)\right| \\
\leq & \varepsilon^{*}\left|\sigma^{\alpha-1}(t)\right|\left(\mu(t)+|s-t|+\mu(t)+\left|\mathbf{T}_{\alpha}\left(f^{\Delta}\right)(t)\right|\right) \\
< & \varepsilon^{*}\left|\sigma^{\alpha-1}(t)\right|\left(1+\left|\mathbf{T}_{\alpha}\left(f^{\Delta}\right)(t)\right|+2 \mu(t)\right) \\
= & \varepsilon .
\end{aligned}
$$

It follows that $f$ is continuous at $t$.

Part (ii). Assume that $f$ is continuous at $t$ and $t$ is right-scattered. By continuity,

$$
\lim _{s \rightarrow t} \frac{f(\sigma(t))-f(s)}{\sigma(t)-s} \sigma^{1-\alpha}(t)=\frac{f(\sigma(t))-f(t)}{\sigma(t)-t} \sigma^{1-\alpha}(t)=\frac{f(\sigma(t))-f(t)}{\mu(t)} \sigma^{1-\alpha}(t) .
$$

Hence, given $\varepsilon>0$, there exists a neighborhood $U$ of $t$ such that

$$
\left|\frac{f(\sigma(t))-f(s)}{\sigma(t)-s} \sigma^{1-\alpha}(t)-\frac{f(\sigma(t))-f(t)}{\mu(t)} \sigma^{1-\alpha}(t)\right| \leq \varepsilon
$$

for all $s \in U$. It follows that

$$
\left|(f(\sigma(t))-f(s)) \sigma^{1-\alpha}(t)-\frac{f(\sigma(t))-f(t)}{\mu(t)}(\sigma(t)-s) \sigma^{1-\alpha}(t)\right| \leq \varepsilon|\sigma(t)-s|
$$

for all $s \in U$. Hence, we get the desired result

$$
\mathbf{T}_{\alpha}\left(f^{\Delta}\right)(t)=\frac{f(\sigma(t))-f(t)}{\mu(t)} \sigma^{1-\alpha}(t) .
$$

Part (iii). Assume that $f$ is conformable $\Delta$ fractional differentiable of order $\alpha$ at $t$ and $t$ is right-dense. Then for each $\varepsilon>0$, there exists a neighborhood $U$ of $t$ such that

$$
\left|(f(\sigma(t))-f(s)) \sigma^{1-\alpha}(t)-\mathbf{T}_{\alpha}\left(f^{\Delta}\right)(t)(\sigma(t)-s)\right| \leq \varepsilon|\sigma(t)-s|
$$

for all $s \in U$. Since $\sigma(t)=t$, we have

$$
\left|(f(t)-f(s)) t^{1-\alpha}-\mathbf{T}_{\alpha}\left(f^{\Delta}\right)(t)(t-s)\right| \leq \varepsilon|t-s|
$$

for all $s \in U$. It follows that

$$
\left|\frac{f(t)-f(s)}{t-s} t^{1-\alpha}-\mathbf{T}_{\alpha}\left(f^{\Delta}\right)(t)\right| \leq \varepsilon
$$

for all $s \in U$ and $s \neq t$. Thus, we conclude that

$$
\mathbf{T}_{\alpha}\left(f^{\Delta}\right)(t)=\lim _{s \rightarrow t} \frac{f(t)-f(s)}{t-s} t^{1-\alpha} .
$$


On the other hand, if the limit

$$
\lim _{s \rightarrow t} \frac{f(t)-f(s)}{t-s} t^{1-\alpha},
$$

exists as a finite number and is equal to $J$, then for each $\varepsilon>0$, there exists a neighborhood $U$ of $t$ such that

$$
\left|(f(t)-f(s)) t^{1-\alpha}-J(t-s)\right| \leq \varepsilon|t-s|
$$

for all $s \in U$. Since $t$ is right-dense, we obtain

$$
\left|(f(\sigma(t))-f(s)) \sigma^{1-\alpha}(t)-J(\sigma(t)-s)\right| \leq \varepsilon|\sigma(t)-s| .
$$

Therefore, $f$ is conformable $\Delta$ fractional differentiable of order $\alpha$ at $t$ and

$$
\mathbf{T}_{\alpha}\left(f^{\Delta}\right)(t)=\lim _{s \rightarrow t} \frac{f(t)-f(s)}{t-s} t^{1-\alpha} .
$$

Part (iv). If $t$ is right-dense, then $\mu(t)=0$ and

$$
f(\sigma(t))=f(t)=f(t)+\mu(t) \mathbf{T}_{\alpha}\left(f^{\Delta}\right)(t) \sigma^{\alpha-1}(t) .
$$

If $t$ is right-scattered, then $\sigma(t)>t$, and by virtue of (ii),

$$
f(\sigma(t))=f(t)+\mu(t) \frac{f(\sigma(t))-f(t)}{\mu(t)}=f(t)+\mu(t) \mathbf{T}_{\alpha}\left(f^{\Delta}\right)(t) \sigma^{\alpha-1}(t) .
$$

The proof is complete.

Example 3.3. We consider the two cases $\mathbb{T}=\mathbb{R}$ and $\mathbb{T}=\mathbb{Z}$.

(i) If $\mathbb{T}=\mathbb{R}$, then $f: \mathbb{R} \rightarrow \mathbb{R}$ is conformable fractional differentiable of order $\alpha$ at $t \in \mathbb{R}$ if and only if the limit

$$
\lim _{s \rightarrow t} \frac{f(t)-f(s)}{t-s} t^{1-\alpha}
$$

exists as a finite number. In this case,

$$
\mathbf{T}_{\alpha}\left(f^{\Delta}\right)(t)=\lim _{s \rightarrow t} \frac{f(t)-f(s)}{t-s} t^{1-\alpha} .
$$

If $\alpha=1$, then

$$
\mathbf{T}_{\alpha}\left(f^{\Delta}\right)(t)=f^{\Delta}(t)=f^{\prime}(t) .
$$

(ii) If $\mathbb{T}=\mathbb{Z}$, then $f: \mathbb{Z} \rightarrow \mathbb{R}$ is conformable $\Delta$ fractional differentiable of order $\alpha$ at $t \in \mathbb{Z}$ with

$$
\mathbf{T}_{\alpha}\left(f^{\Delta}\right)(t)=\frac{f(t+1)-f(t)}{1} f^{1-\alpha}(t+1)=f^{1-\alpha}(t+1)(f(t+1)-f(t)) .
$$

If $\alpha=1$, then

$$
\mathbf{T}_{\alpha}\left(f^{\Delta}\right)(t)=f(t+1)-f(t)=\Delta f(t),
$$

where $\Delta$ is the usual forward difference operator.

\section{Example 3.4.}

(i) If $f: \mathbb{T} \rightarrow \mathbb{R}$ is defined by $f(t)=C$ for all $t \in \mathbb{T}$, where $C \in \mathbb{R}$ is a constant, then $\mathbf{T}_{\alpha}\left(f^{\Delta}\right)(t) \equiv 0$.

This is clear because for any $\varepsilon>0$,

$$
\left|(f(\sigma(t))-f(s)) \sigma^{1-\alpha}(t)-0 \cdot(\sigma(t)-s)\right|=\left|(C-C) \sigma^{1-\alpha}(t)\right|=0 \leq \varepsilon|\sigma(t)-s| 1
$$

holds for all $s \in \mathbb{T}$. 
(ii) If $f: \mathbb{T} \rightarrow \mathbb{R}$ is defined by $f(t)=t$ for all $t \in \mathbb{T}$, then $\mathbf{T}_{\alpha}\left(f^{\Delta}\right)(t)=\sigma^{1-\alpha}(t)$. This is valid because for any $\varepsilon>0$,

$$
\begin{aligned}
\left|(f(\sigma(t))-f(s)) \sigma^{1-\alpha}(t)-\sigma^{1-\alpha}(t)(\sigma(t)-s)\right| & =\left|(\sigma(t)-s) \sigma^{1-\alpha}(t)-\sigma^{1-\alpha}(t)(\sigma(t)-s)\right| \\
& =0 \leq \varepsilon|\sigma(t)-s|
\end{aligned}
$$

holds for all $s \in \mathbb{T}$.

If $\alpha=1$, then $\mathbf{T}_{\alpha}\left(f^{\Delta}\right)(t) \equiv 1$.

Example 3.5. Suppose that $f: \mathbb{T} \rightarrow \mathbb{R}$ is defined by $f(t)=t^{2}$ for all $t \in \mathbb{T}:=\left\{n / 2: n \in \mathbb{N}_{0}\right\}$. By virtue of Theorem 3.2 (ii), we have that $f$ is conformable $\Delta$ fractional differentiable of order $\alpha$ at $t \in \mathbb{T}$ with

$$
\mathbf{T}_{\alpha}\left(f^{\Delta}\right)(t)=\left(2 t+\frac{1}{2}\right)\left(t+\frac{1}{2}\right)^{1-\alpha} .
$$

Theorem 3.6. Assume $f, g: \mathbb{T} \rightarrow \mathbb{R}$ are conformable $\Delta$ fractional differentiable of order $\alpha$ at $t \in \mathbb{T}^{k}$. Then we have the following.

(i) For all constants $\lambda_{1}$ and $\lambda_{2}$, the sum $\lambda_{1} f+\lambda_{2} g: \mathbb{T} \rightarrow \mathbb{R}$ is conformable $\Delta$ fractional differentiable of order $\alpha$ at $t \in \mathbb{T}^{k}$ with

$$
\mathbf{T}_{\alpha}\left(\left(\lambda_{1} f+\lambda_{2} g\right)^{\Delta}\right)(t)=\lambda_{1} \mathbf{T}_{\alpha}\left(f^{\Delta}\right)(t)+\lambda_{2} \mathbf{T}_{\alpha}\left(g^{\Delta}\right)(t)
$$

(ii) The product $f g: \mathbb{T} \rightarrow \mathbb{R}$ is conformable $\Delta$ fractional differentiable of order $\alpha$ at $t$ with

$$
\mathbf{T}_{\alpha}\left((f g)^{\Delta}\right)(t)=\mathbf{T}_{\alpha}\left(f^{\Delta}\right)(t) g(t)+f(\sigma(t)) \mathbf{T}_{\alpha}\left(g^{\Delta}\right)(t)=f(t) \mathbf{T}_{\alpha}\left(g^{\Delta}\right)(t)+\mathbf{T}_{\alpha}\left(f^{\Delta}\right)(t) g(\sigma(t)) .
$$

(iii) If $f(t) f(\sigma(t)) \neq 0$, then $1 / f$ is conformable $\Delta$ fractional differentiable of order $\alpha$ at $t$ with

$$
\mathbf{T}_{\alpha}\left(\left(\frac{1}{f}\right)^{\Delta}\right)(t)=-\frac{\mathbf{T}_{\alpha}\left(f^{\Delta}\right)(t)}{f(t) f(\sigma(t))}
$$

(iv) If $g(t) g(\sigma(t)) \neq 0$, then $\mathrm{f} / \mathrm{g}$ is conformable $\Delta$ fractional differentiable of order $\alpha$ at $t$ with

$$
\mathbf{T}_{\alpha}\left(\left(\frac{f}{g}\right)^{\Delta}\right)(t)=\frac{\mathbf{T}_{\alpha}\left(f^{\Delta}\right)(t) g(t)-f(t) \mathbf{T}_{\alpha}\left(g^{\Delta}\right)(t)}{g(t) g(\sigma(t))} .
$$

Proof. Part (i). Let $\varepsilon>0$. Then there exist neighborhoods $U_{1}$ and $U_{2}$ of $t$ such that

$$
\left|\left(\lambda_{1} f(\sigma(t))-\lambda_{1} f(s)\right) \sigma^{1-\alpha}(t)-\lambda_{1} \mathbf{T}_{\alpha}\left(f^{\Delta}\right)(t)(\sigma(t)-s)\right| \leq \frac{\varepsilon}{2}\left|\lambda_{1}(\sigma(t)-s)\right|
$$

for all $s \in U_{1}$ and

$$
\left|\left(\lambda_{2} g(\sigma(t))-\lambda_{2} g(s)\right) \sigma^{1-\alpha}(t)-\lambda_{2} \mathbf{T}_{\alpha}\left(g^{\Delta}\right)(t)(\sigma(t)-s)\right| \leq \frac{\varepsilon}{2}\left|\lambda_{2}(\sigma(t)-s)\right|
$$

for all $s \in U_{2}$. Let $U:=U_{1} \cap U_{2}$ and $\lambda:=\max \left\{\lambda_{1}, \lambda_{2}\right\}$. Then, we have, for all $s \in U$,

$$
\left|\left(\left(\lambda_{1} f+\lambda_{2} g\right)(\sigma(t))-\left(\lambda_{1} f+\lambda_{2} g\right)(s)\right) \sigma^{1-\alpha}(t)-\left(\lambda_{1} \mathbf{T}_{\alpha}\left(f^{\Delta}\right)(t)+\lambda_{2} \mathbf{T}_{\alpha}\left(g^{\Delta}\right)(t)\right)(\sigma(t)-s)\right|
$$




$$
\begin{aligned}
\leq & \left|\left(\lambda_{1} f(\sigma(t))-\lambda_{1} f(s)\right) \sigma^{1-\alpha}(t)-\lambda_{1} \mathbf{T}_{\alpha}\left(f^{\Delta}\right)(t)(\sigma(t)-s)\right| \\
& +\left|\left(\lambda_{2} g(\sigma(t))-\lambda_{2} g(s)\right) \sigma^{1-\alpha}(t)-\lambda_{2} \mathbf{T}_{\alpha}\left(g^{\Delta}\right)(t)(\sigma(t)-s)\right| \\
\leq & \frac{\varepsilon}{2}\left|\lambda_{1}(\sigma(t)-s)\right|+\frac{\varepsilon}{2}\left|\lambda_{2}(\sigma(t)-s)\right| \leq \varepsilon|\lambda(\sigma(t)-s)| .
\end{aligned}
$$

Therefore, $\lambda_{1} f+\lambda_{2} g$ is conformable $\Delta$ fractional differentiable of order $\alpha$ at $t \in \mathbb{T}^{k}$ with

$$
\mathbf{T}_{\alpha}\left(\left(\lambda_{1} f+\lambda_{2} g\right)^{\Delta}\right)(t)=\lambda_{1} \mathbf{T}_{\alpha}\left(f^{\Delta}\right)(t)+\lambda_{2} \mathbf{T}_{\alpha}\left(g^{\Delta}\right)(t) .
$$

Part (ii). Let $0<\varepsilon<1$. Define

$$
\varepsilon^{*}:=\frac{\varepsilon}{1+|g(\sigma(t))|+|f(t)|+\left|\mathbf{T}_{\alpha}\left(g^{\Delta}\right)(t)\right|} .
$$

Then $0<\varepsilon^{*}<1$. Since $f, g: \mathbb{T} \rightarrow \mathbb{R}$ are conformable $\Delta$ fractional differentiable of order $\alpha$ at $t \in \mathbb{T}^{k}$, there exist neighborhoods $U_{1}$ and $U_{2}$ of $t$ such that

$$
\left|(f(\sigma(t))-f(s)) \sigma^{1-\alpha}(t)-\mathbf{T}_{\alpha}\left(f^{\Delta}\right)(t)(\sigma(t)-s)\right| \leq \varepsilon^{*}|\sigma(t)-s|
$$

for all $s \in U_{1}$ and

$$
\left|(g(\sigma(t))-g(s)) \sigma^{1-\alpha}(t)-\mathbf{T}_{\alpha}\left(g^{\Delta}\right)(t)(\sigma(t)-s)\right| \leq \varepsilon^{*}|\sigma(t)-s|
$$

for all $s \in U_{2}$. From Theorem 3.2 (i), there exists a neighborhood $U_{3}$ of $t$ such that

$$
|f(t)-f(s)| \leq \varepsilon^{*}
$$

for all $s \in U_{3}$. Let $U:=U_{1} \cap U_{2} \cap U_{3}$. Then, we have, for all $s \in U$,

$$
\begin{aligned}
&\left|[f(\sigma(t)) g(\sigma(t))-f(s) g(s)] \sigma^{1-\alpha}(t)-\left[\mathbf{T}_{\alpha}\left(f^{\Delta}\right)(t) g(\sigma(t))+f(t) \mathbf{T}_{\alpha}\left(g^{\Delta}\right)(t)\right](\sigma(t)-s)\right| \\
& \leq\left|\left[(f(\sigma(t))-f(s)) \sigma^{1-\alpha}(t)-\mathbf{T}_{\alpha}\left(f^{\Delta}\right)(t)(\sigma(t)-s)\right] g(\sigma(t))\right| \\
&+\left|\left[(g(\sigma(t))-g(s)) \sigma^{1-\alpha}(t)-\mathbf{T}_{\alpha}\left(g^{\Delta}\right)(t)(\sigma(t)-s)\right] f(t)\right| \\
&+\left|\left[(g(\sigma(t))-g(s)) \sigma^{1-\alpha}(t)-\mathbf{T}_{\alpha}\left(g^{\Delta}\right)(t)(\sigma(t)-s)\right](f(s)-f(t))\right| \\
&+\left|\mathbf{T}_{\alpha}\left(g^{\Delta}\right)(t)(\sigma(t)-s)(f(s)-f(t))\right| \\
& \leq \varepsilon^{*}|\sigma(t)-s|\left(|g(\sigma(t))|+|f(t)|+\varepsilon^{*}+\left|\mathbf{T}_{\alpha}\left(g^{\Delta}\right)(t)\right|\right) \\
& \leq \varepsilon|\sigma(t)-s| .
\end{aligned}
$$

Thus, we deduce that

$$
\mathbf{T}_{\alpha}\left((f g)^{\Delta}\right)(t)=f(t) \mathbf{T}_{\alpha}\left(g^{\Delta}\right)(t)+\mathbf{T}_{\alpha}\left(f^{\Delta}\right)(t) g(\sigma(t)) .
$$

One can easily obtain another product rule by interchanging the role of $f$ and $g$.

Part (iii). From Example 3.4 .

$$
\mathbf{T}_{\alpha}\left(\left(f \cdot \frac{1}{f}\right)^{\Delta}\right)(t)=\mathbf{T}_{\alpha}\left((1)^{\Delta}\right)(t)=0 .
$$

Therefore,

$$
\mathbf{T}_{\alpha}\left(\left(\frac{1}{f}\right)^{\Delta}\right)(t) f(\sigma(t))+\mathbf{T}_{\alpha}\left(f^{\Delta}\right)(t) \frac{1}{f(t)}=0
$$


and consequently

$$
\mathbf{T}_{\alpha}\left(\left(\frac{1}{f}\right)^{\Delta}\right)(t)=-\frac{\mathbf{T}_{\alpha}\left(f^{\Delta}\right)(t)}{f(t) f(\sigma(t))} .
$$

Part (iv). Applications of (ii) and (iii) imply that

$$
\begin{aligned}
\mathbf{T}_{\alpha}\left(\left(\frac{f}{g}\right)^{\Delta}\right)(t) & =f(t) \mathbf{T}_{\alpha}\left(\left(\frac{1}{g}\right)^{\Delta}\right)(t)+\mathbf{T}_{\alpha}\left(f^{\Delta}\right)(t) \frac{1}{g(\sigma(t))} \\
& =-f(t) \frac{\mathbf{T}_{\alpha}\left(g^{\Delta}\right)(t)}{g(t) g(\sigma(t))}+\mathbf{T}_{\alpha}\left(f^{\Delta}\right)(t) \frac{1}{g(\sigma(t))} \\
& =\frac{\mathbf{T}_{\alpha}\left(f^{\Delta}\right)(t) g(t)-f(t) \mathbf{T}_{\alpha}\left(g^{\Delta}\right)(t)}{g(t) g(\sigma(t))}
\end{aligned}
$$

The proof is complete.

Theorem 3.7. Let $c$ be a constant and $m \in \mathbb{N}_{+}:=\{1,2, \ldots\}$.

(i) If $f(t)=(t-c)^{m}$, then

$$
\mathbf{T}_{\alpha}\left(f^{\Delta}\right)(t)=\sigma^{1-\alpha}(t) \sum_{i=0}^{m-1}(\sigma(t)-c)^{i}(t-c)^{m-1-i} .
$$

(ii) If $g(t)=1 / f(t)=1 /(t-c)^{m}$, then

$$
\mathbf{T}_{\alpha}\left(g^{\Delta}\right)(t)=-\sigma^{1-\alpha}(t) \sum_{i=0}^{m-1} \frac{1}{(\sigma(t)-c)^{m-i}(t-c)^{i+1}},
$$

provided $(\sigma(t)-c)(t-c) \neq 0$.

Proof. Part (i). We prove the first formula by induction. If $m=1$, then $f(t)=t-c$, and $\mathbf{T}_{\alpha}\left(f^{\Delta}\right)(t)=$ $\sigma^{1-\alpha}(t)$ holds when using Example 3.4 and Theorem 3.6 (i). We assume now that

$$
\mathbf{T}_{\alpha}\left(f^{\Delta}\right)(t)=\sigma^{1-\alpha}(t) \sum_{i=0}^{m-1}(\sigma(t)-c)^{i}(t-c)^{m-1-i}
$$

holds for $f(t)=(t-c)^{m}$ and let $F(t):=(t-c)^{m+1}=(t-c) f(t)$. An application of Theorem 3.6 (ii) yields

$$
\begin{aligned}
\mathbf{T}_{\alpha}(F)^{\Delta}(t) & =\sigma^{1-\alpha}(t) f(\sigma(t))+(t-c) \mathbf{T}_{\alpha}\left(f^{\Delta}\right)(t) \\
& =\sigma^{1-\alpha}(t)(\sigma(t)-c)^{m}+(t-c) \sigma^{1-\alpha}(t) \sum_{i=0}^{m-1}(\sigma(t)-c)^{i}(t-c)^{m-1-i} \\
& =\sigma^{1-\alpha}(t) \sum_{i=0}^{m}(\sigma(t)-c)^{i}(t-c)^{m-i}
\end{aligned}
$$

Hence, part (i) is intact. 
Part (ii). For $g(t)=1 /(t-c)^{m}=1 / f(t)$, we use Theorem 3.6 (iii) to arrive at

$$
\begin{aligned}
\mathbf{T}_{\alpha}(g)^{\Delta}(t) & =-\frac{\mathbf{T}_{\alpha}\left(f^{\Delta}\right)(t)}{f(t) f(\sigma(t))} \\
& =-\frac{\sigma^{1-\alpha}(t) \sum_{i=0}^{m-1}(\sigma(t)-c)^{i}(t-c)^{m-1-i}}{(\sigma(t)-c)^{m}(t-c)^{m}} \\
& =-\sigma^{1-\alpha}(t) \sum_{i=0}^{m-1} \frac{1}{(\sigma(t)-c)^{m-i}(t-c)^{i+1}},
\end{aligned}
$$

provided $(\sigma(t)-c)(t-c) \neq 0$. This completes the proof.

Example 3.8. If $f: \mathbb{T} \rightarrow \mathbb{R}$ is defined by $f(t)=1 / t^{2}$ for all $t \in \mathbb{T}:=\left\{\sqrt{n}: n \in \mathbb{N}_{0}\right\}$, then we have that $f$ is conformable $\Delta$ fractional differentiable of order $\alpha$ at $t \in \mathbb{T}$ with

$$
\mathbf{T}_{\alpha}\left(f^{\Delta}\right)(t)=-\sigma^{1-\alpha}(t)\left(\frac{1}{t \sigma^{2}(t)}+\frac{1}{t^{2} \sigma(t)}\right)=-\left(\sqrt{t^{2}+1}\right)^{-\alpha}\left(\frac{1}{t \sqrt{t^{2}+1}}+\frac{1}{t^{2}}\right) .
$$

\section{Conformable delta fractional integration}

Definition 4.1. Assume $f: \mathbb{T} \rightarrow \mathbb{R}$ is a regulated function. We define the indefinite $\alpha$-conformable $\Delta$ fractional integral of $f$ by

$$
\mathbf{I}_{\alpha}\left(f^{\Delta}\right)(t)+C=\int f(t) \Delta_{\alpha} t=\int f(t) \sigma^{\alpha-1}(t) \Delta t
$$

where $C$ is an arbitrary constant. $\mathbf{I}_{\alpha}\left(f^{\Delta}\right)(t)$ is called a pre-antiderivative of $f$. We define the Cauchy $\alpha$-conformable $\Delta$ fractional integral by

$$
\int_{a}^{b} f(t) \Delta_{\alpha} t=\mathbf{I}_{\alpha}\left(f^{\Delta}\right)(b)-\mathbf{I}_{\alpha}\left(f^{\Delta}\right)(a)
$$

for all $a, b \in \mathbb{T}$. A function $\mathbf{I}_{\alpha}\left(f^{\Delta}\right): \mathbb{T} \rightarrow \mathbb{R}$ is called an antiderivative of $f: \mathbb{T} \rightarrow \mathbb{R}$ provided

$$
\left(\mathbf{T}_{\alpha} \mathbf{I}_{\alpha}\left(f^{\Delta}\right)\right)(t)=f(t)
$$

for all $t \in \mathbb{T}^{k}$.

Theorem 4.2 (Existence of antiderivatives). For every $r d$-continuous function $f: \mathbb{T} \rightarrow \mathbb{R}$, there exists a function $\mathbf{I}_{\alpha}\left(f^{\Delta}\right)$ such that

$$
\left(\mathbf{T}_{\alpha} \mathbf{I}_{\alpha}\left(f^{\Delta}\right)\right)(t)=f(t) .
$$

Proof. Suppose $f: \mathbb{T} \rightarrow \mathbb{R}$ is rd-continuous. By [16, Theorem 1.60], $f$ is regulated. Similar to the proof of [16, Theorem 8.13], we conclude that $\mathbf{I}_{\alpha}\left(f^{\Delta}\right)$ is conformable $\Delta$ fractional differentiable of order $\alpha$ at $t \in \mathbb{T}^{k}$. Then

$$
\left(\mathbf{T}_{\alpha} \mathbf{I}_{\alpha}\left(f^{\Delta}\right)\right)(t)=\sigma^{1-\alpha}(t) \sigma^{\alpha-1}(t) f(t)=f(t) .
$$

This completes the proof.

We can easily get the following theorem. 
Theorem 4.3. Let $a, b, c \in \mathbb{T}, \lambda_{1}, \lambda_{2} \in \mathbb{R}$, and $f, g: \mathbb{T} \rightarrow \mathbb{R}$ be rd-continuous functions. Then

(i) $\int_{a}^{b}\left(\lambda_{1} f(t)+\lambda_{2} g(t)\right) \Delta_{\alpha} t=\lambda_{1} \int_{a}^{b} f(t) \Delta_{\alpha} t+\lambda_{2} \int_{a}^{b} g(t) \Delta_{\alpha} t$;

(ii) $\int_{a}^{b} f(t) \Delta_{\alpha} t=-\int_{b}^{a} f(t) \Delta_{\alpha} t$;

(iii) $\int_{a}^{b} f(t) \Delta_{\alpha} t=\int_{a}^{c} f(t) \Delta_{\alpha} t+\int_{c}^{b} f(t) \Delta_{\alpha} t$;

(iv) $\int_{a}^{b} f(\sigma(t)) \mathbf{T}_{\alpha}\left(g^{\Delta}\right)(t) \Delta_{\alpha} t=f(b) g(b)-f(a) g(a)-\int_{a}^{b} \mathbf{T}_{\alpha}\left(f^{\Delta}\right)(t) g(t) \Delta_{\alpha} t$;

(v) $\int_{a}^{b} f(t) \mathbf{T}_{\alpha}\left(g^{\Delta}\right)(t) \Delta_{\alpha} t=f(b) g(b)-f(a) g(a)-\int_{a}^{b} \mathbf{T}_{\alpha}\left(f^{\Delta}\right)(t) g(\sigma(t)) \Delta_{\alpha} t ;$

(vi) $\int_{a}^{a} f(t) \Delta_{\alpha} t=0$;

(vii) if $|f(t)| \leq g(t)$ on $[a, b)_{\mathbb{T}}$, then $\left|\int_{a}^{b} f(t) \Delta_{\alpha} t\right| \leq \int_{a}^{b} g(t) \Delta_{\alpha} t$;

(viii) if $f(t) \geq 0$ for all $t \in[a, b)_{\mathbb{T}}$, then $\int_{a}^{b} f(t) \Delta_{\alpha} t \geq 0$.

Theorem 4.4. Let $a, b \in \mathbb{T}$, and let $f: \mathbb{T} \rightarrow \mathbb{R}$ be an rd-continuous function. Then we have the following.

(i) If $\mathbb{T}=\mathbb{R}$, then $\int_{a}^{b} f(t) \Delta_{\alpha} t=\int_{a}^{b} f(t) / t^{1-\alpha} d t$, where the integral on the right is the conformable fractional integral given in [23]. If $\alpha=1$, then it reduces to the usual Riemann integral.

(ii) If $[a, b]_{\mathbb{T}}$ consists of only isolated points, then

$$
\int_{a}^{b} f(t) \Delta_{\alpha} t= \begin{cases}\sum_{t \in[a, b)_{\mathbb{T}}} \sigma^{\alpha-1}(t) \mu(t) f(t) & \text { if } a<b \\ 0 & \text { if } a=b, \\ -\sum_{t \in[b, a)_{\mathbb{T}}} \sigma^{\alpha-1}(t) \mu(t) f(t) & \text { if } a>b .\end{cases}
$$

(iii) If $\mathbb{T}=h \mathbb{Z}=\{h k: k \in \mathbb{Z}\}$, where $h>0$, then

$$
\int_{a}^{b} f(t) \Delta_{\alpha} t= \begin{cases}\sum_{k=\frac{a}{h}}^{\frac{b}{h}-1}(h k+h)^{\alpha-1} h f(h k) & \text { if } a<b, \\ 0 & \text { if } a=b, \\ -\sum_{k=\frac{b}{h}}^{\frac{a}{h}-1}(h k+h)^{\alpha-1} h f(h k) & \text { if } a>b .\end{cases}
$$

(iv) If $\mathbb{T}=\mathbb{Z}$, then

$$
\int_{a}^{b} f(t) \Delta_{\alpha} t= \begin{cases}\sum_{t=a}^{b-1}(t+1)^{\alpha-1} f(t) & \text { if } a<b \\ 0 & \text { if } a=b \\ -\sum_{t=b}^{a-1}(t+1)^{\alpha-1} f(t) & \text { if } a>b\end{cases}
$$

Proof. Part (i). The proof is not difficult and so is omitted.

Part (ii). First, note that $[a, b]_{\mathbb{T}}$ consists of only finitely isolated points. Assume that $a<b$ and $[a, b]_{\mathbb{T}}=\left\{t_{0}, t_{1}, \ldots, t_{n}\right\}$, where

$$
a=t_{0}<t_{1}<t_{2}<\ldots<t_{n}=b .
$$


By virtue of Theorem 4.3 (iii),

$$
\int_{a}^{b} f(t) \Delta_{\alpha} t=\sum_{i=0}^{n-1} \int_{t_{i}}^{t_{i+1}} f(t) \Delta_{\alpha} t=\sum_{i=0}^{n-1} \int_{t_{i}}^{\sigma\left(t_{i}\right)} f(t) \Delta_{\alpha} t=\sum_{i=0}^{n-1} \mu\left(t_{i}\right) \sigma^{\alpha-1}\left(t_{i}\right) f\left(t_{i}\right) .
$$

Consequently,

$$
\int_{a}^{b} f(t) \Delta_{\alpha} t=\sum_{t \in[a, b)_{\mathbb{T}}} \sigma^{\alpha-1}(t) \mu(t) f(t)
$$

If $a>b$, then the result follows from what we just proved and Theorem 4.3 (ii). If $a=b$, then the result follows from Theorem 4.3 (vi).

Part (iii) and (iv) are special cases of Part (ii). The proof is complete.

Example 4.5. If $f: \mathbb{T} \rightarrow \mathbb{R}$ is defined by $f(t)=t^{3}$ for all $\mathbb{T}=\mathbb{R}$ and $\alpha=1 / 2$, then

$$
\int_{1}^{4} f(t) \Delta_{\alpha} t=\int_{1}^{4} t^{3} \Delta_{\frac{1}{2}} t=\int_{1}^{4} t^{3} \Delta_{\frac{1}{2}} t=\int_{1}^{4} t^{\frac{5}{2}} d t=36 \frac{2}{7}
$$

Example 4.6. If $f: \mathbb{T} \rightarrow \mathbb{R}$ is defined by $f(t)=t^{2}$ for all $t \in \mathbb{T}:=\left\{n / 2: n \in \mathbb{N}_{0}\right\}$ and $\alpha=1 / 2$, then

$$
\begin{aligned}
\int_{1}^{3} f(t) \Delta_{\alpha} t & =\int_{1}^{3} t^{2} \Delta_{\frac{1}{2}} t \\
& =\sqrt{\frac{2}{3}} \cdot \frac{1}{2} \cdot 1^{2}+\sqrt{\frac{2}{4}} \cdot \frac{1}{2} \cdot\left(\frac{3}{2}\right)^{2}+\sqrt{\frac{2}{5}} \cdot \frac{1}{2} \cdot\left(\frac{4}{2}\right)^{2}+\sqrt{\frac{2}{6}} \cdot \frac{1}{2} \cdot\left(\frac{5}{2}\right)^{2} \\
& =\frac{\sqrt{6}}{6}+\frac{9 \sqrt{2}}{16}+\frac{2 \sqrt{10}}{5}+\frac{25 \sqrt{3}}{24}
\end{aligned}
$$

\section{Conclusions}

This paper investigated the conformable delta fractional calculus on time scales. The results of the paper give a common generalization of the conformable fractional derivative and the usual delta derivative. Another interesting line of research is to investigate conformable fractional derivative on time scales in other different directions rather than the one considered here. For instance, instead of following the delta approach that we have adopted, one can develop a nabla, a diamond, or a symmetric time scale fractional calculus. These problems will be subject of future research.

\section{Acknowledgements}

This research was supported by NNSF of P. R. China (Grant Nos. 61503171, 61403061, and 11447005), CPSF (Grant No. 2015M582091), NSF of Shandong Province (Grant No. ZR2012FL06), Educational Commission of Hubei Province (Grant No. Q20152505), DSRF of Linyi University (Grant No. LYDX2015BS001), and the AMEP of Linyi University, P. R. China.

\section{References}

[1] T. Abdeljawad, On conformable fractional calculus, J. Comput. Appl. Math., 279 (2015), 57-66. 1

[2] T. Abdeljawad, M. Al Horani, R. Khalil, Conformable fractional semigroups of operators, J. Semigroup Theory Appl., 2015 (2015), 9 pages.

[3] I. Abu Hammad, R. Khalil, Fractional Fourier series with applications, Amer. J. Comput. Appl. Math., 4 (2014), 187-191. 
[4] M. Abu Hammad, R. Khalil, Abel's formula and Wronskian for conformable fractional differential equations, Int. J. Differ. Equ. Appl., 13 (2014), 177-183.

[5] M. Abu Hammad, R. Khalil, Conformable fractional heat differential equations, Int. J. Pure. Appl. Math., 94 (2014), 215-221.

[6] M. Abu Hammad, R. Khalil, Legendre fractional differential equation and Legendre fractional polynomials, Int. J. Appl. Math. Res., 3 (2014), 214-219. 1

[7] R. P. Agarwal, M. Bohner, T. Li, Oscillatory behavior of second-order half-linear damped dynamic equations, Appl. Math. Comput., 254 (2015), 408-418. 1

[8] M. Al Horani, M. Abu Hammad, R. Khalil, Variation of parameters for local fractional nonhomogeneous linear-differential equations, J. Math. Computer Sci., 16 (2016), 147-153. 1

[9] H. Batarfi, J. Losada, J. J. Nieto, W. Shammakh, Three-point boundary value problems for conformable fractional differential equations, J. Funct. Spaces, 2015 (2015), 6 pages. 1

[10] B. Bayour, D. F. M. Torres, Existence of solution to a local fractional nonlinear differential equation, J. Comput. Appl. Math., 2016 (2016), (in press). 1

[11] N. Benkhettou, A. M. C. Brito da Cruz, D. F. M. Torres, A fractional calculus on arbitrary time scales: Fractional differentiation and fractional integration, Signal Process., 107 (2015), 230-237. 1 .

[12] N. Benkhettou, A. M. C. Brito da Cruz, D. F. M. Torres, Nonsymmetric and symmetric fractional calculi on arbitrary nonempty closed sets, Math. Methods Appl. Sci., 39 (2016), 261-279. 1

[13] N. Benkhettou, S. Hassani, D. F. M. Torres, A conformable fractional calculus on arbitrary time scales, J. King Saud Univ. Sci., 28 (2016), 93-98. 1, 3

[14] M. Bohner, T. Li, Oscillation of second-order p-Laplace dynamic equations with a nonpositive neutral coefficient, Appl. Math. Lett., 37 (2014), 72-76. 1

[15] M. J. Bohner, R. R. Mahmoud, S. H. Saker, Discrete, continuous, delta, nabla, and diamond-alpha Opial inequalities, Math. Inequal. Appl., 18 (2015), 923-940.

[16] M. Bohner, A. Peterson, Dynamic Equations on Time Scales: An Introduction with Application, Birkhäuser, Boston, (2001). 4

[17] M. Bohner, A. Peterson, Advances in Dynamic Equations on Time Scales, Birkhäuser, Boston, (2003).

[18] M. Bohner, S. H. Saker, Sneak-out principle on time scales, J. Math. Inequal., 10 (2016), 393-403. 1

[19] A. Carpinteri, F. Mainardi, Fractals and Fractional Calculus in Continuum Mechanics, Springer-Verlag, Vienna, (1997). 1

[20] R. Herrmann, Fractional Calculus: An Introduction for Physicists, World Scientific, Singapore, (2011). 1.

[21] S. Hilger, Eın Maßkettenkalkül mıt Anwendung auf Zentrumsmannıgaltıgkerten, Ph.D. Thesis, Universtät Würzburg, (1988). 1

[22] R. Khalil, M. Al Horani, D. Anderson, Undetermined coefficients for local fractional differential equations, J. Math. Computer Sci., 16 (2016), 140-146. 1

[23] R. Khalil, M. Al Horani, A. Yousef, M. Sababheh, A new definition of fractional derivative, J. Comput. Appl. Math., 264 (2014), 65-70. 1, 4.4

[24] T. Li, J. Diblík, A. Domoshnitsky, Yu. V. Rogovchenko, F. Sadyrbaev, Q.-R. Wang, Qualitative analysis of differential, difference equations, and dynamic equations on time scales, Abstr. Appl. Anal., 2015 (2015), 3 pages. 1

[25] T. Li, S. H. Saker, A note on oscillation criteria for second-order neutral dynamic equations on isolated time scales, Commun. Nonlinear Sci. Numer. Simul., 19 (2014), 4185-4188. 1

[26] K. S. Miller, B. Ross, An Introduction to the Fractional Calculus and Fractional Differential Equations, John Wiley \& Sons, Inc., New York, (1993). 1

[27] K. B. Oldham, J. Spanier, The Fractional Calculus: Theory and Applications of Differentiation and Integration to Arbitrary Order, Dover Publications, New York-London, (2006). 1

[28] M. D. Ortigueira, Fractional Calculus for Scientists and Engineers, Springer, Dordrecht, (2011). 1]

[29] A. Peterson, B. Thompson, Henstock-Kurzweil delta and nabla integrals, J. Math. Anal. Appl., 323 (2006), 162-178. 1

[30] I. Podlubny, Fractional Differential Equations, Academic Press, San Diego, (1999). 1

[31] J. Sabatier, O. P. Agrawal, J. A. T. Machado, Advances in Fractional Calculus: Theoretical Developments and Applications in Physics and Engineering, Springer, Dordrecht, (2007). 1

[32] S. G. Samko, A. A. Kilbas, O. I. Marichev, Fractional Integrals and Derivatives: Theory and Applications, CRC Press, Switzerland, (1993). 1 